\title{
Retroperitoneal Cystic Lymphangioma: Case Report
}

\author{
S. A. Fanaei ${ }^{1}$, S. A. Ziaee ${ }^{2}$ \\ ${ }^{1}$ Baqiyatallah University of Medical Science, Tehran, Iran \\ ${ }^{2}$ Clinical research Unit, Emergency Department, Atieh Hospital, Tehran, Iran \\ E-mail:Sali_ziaee@yahoo.com \\ Received January 8, 2011; revised March 30, 2011; accepted May 9, 2011.
}

\begin{abstract}
Introduction: Retroperitoneal cystic lymphangioma is a rare benign tumor of the retroperitoneal lymphatics that usually manifests in infancy. It is worth to Report of unexpected presentation especially in Adult. Case Presentation: An adult patient with Left Lower abdominal pain was referred to our hospital. Abdominal ultrasonography revealed a large, multilocular, cystic mass with an obscure margin. CT of the abdomen showed a large homogeneous mass. Laparoscopy revealed a huge retroperitoneal cystic lymphangioma confirmed by histopathology. Conclusions: Adult cyst lymphangioma is the rare benign tumor with unclear intra abdominal manifestation. In our report the retroperitoneum was the rare location of this tumor.
\end{abstract}

Keywords: Lymphangioma, Cystic, Retroperitoneal

\section{Background}

One of the developmental malformations of lymphatic tissue is cystic lymphangioma. It is essentially a malformation of one of the abdominal lymph vessels where a portion is dilated and forms a lymph fluid-filled cyst. Symptoms may vary depending on the exact location and size of the cyst. Cystic lymphangioma is a rare benign tumor. It usually manifests in infancy in head and neck [1]. We report a rare case of adult retroperitoneal lymphangioma.

\section{Case Report}

A 26-year-old Female patient with a mass in left inguinal fossa and left lower abdominal pain was referred to our hospital. Her family and Gynecological and medical history were unremarkable. She had no history of previous abdominal surgery. On physical examination, a soft, non-tender mass was palpated in the left lower abdomen. Auscultation of the abdomen revealed normal bowel sounds. Laboratory data including tumor markers were within normal limits. A plain film of the abdomen showed several loops of small intestine without dilatation or air-fluid level. Abdominal ultrasonography revealed a large, multilocular, cystic mass with an obscure margin. CT of the abdomen showed a large homogeneous mass measuring $14 \mathrm{~cm} \times 6 \mathrm{~cm} \times 5 \mathrm{~cm}$ in size with multiple enhancement septa by contrast medium. This lesion was located near the descending colon, causing compression and stretching of the bowel.

Preoperative diagnosis based on imaging investigations could not be made with any certainty and a percutaneous biopsy of the lesion was deemed too high-risk due to the location and the possibility of malignant seeding if the lesion was neoplastic.

A laparoscopy was ultimately performed. A large retroperitoneal multicystic tumour attached descending colon was identified. The cyst was excised intact. The histopathological examination of the cyst wall demonstrated fibrosis, inflammatory cell infiltrate and the presence of endothelial lined vascular channels. (Figure 1) These findings confirmed a benign lymphangiomatous cyst and a diagnosis of retroperitoneal cystic lymphangioma. Recovery of the patient was uncomplicated.

Although macroscopically, the resection was complete, lymphangioma recurred during a follow up period in this case with chylascos presentation. (Figure 2)

\section{Discussion}

The differential diagnosis of cystic tumour in the retroperitoneum raises several possibilities. These include both malignant and benign tumours. Malignant causes include necrotic neoplasms, germ cell tumours (teratoma), undifferentiated sarcoma, cystic metastases (gastric/ovarian), 


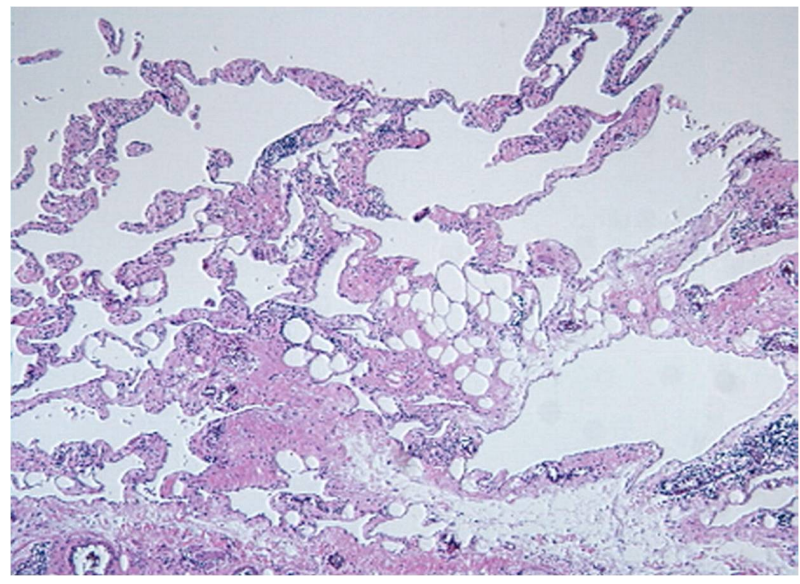

Figure 1. Retroperitoneal cystic lymphangioma in a 24year-old woman. Photomicrograph (original magnification, $\times 40$; hematoxylin-eosin [H-E] stain) shows variable-sized thin-walled cystic spaces in the stroma that are lined with endothelial cells and contain lymphoid aggregation.

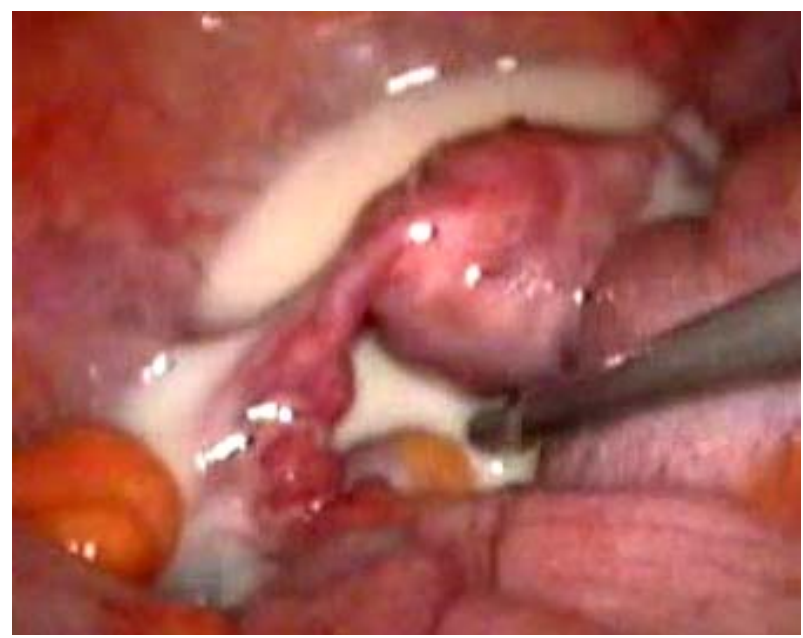

Figure 2. laparoscopic excision of retroperitoneal cyst lymphangioma.

malignant mesenchymoma, biliary cystadenoma/carcinoma and cystic mesothelioma [1]. Benign cystic lesions of the retroperitoneum include lymphangioma, microcystic pancreatic adenoma and cysts of urothelial and foregut origin. Cysts of foregut origin are subdivided into bronchogenic cysts, which contain cartilage or seromucinous respiratory glands, oesophageal cysts, which are composed of well-developed layers of smooth muscle without cartilage, and simple foregut cysts, which have none of these distinguishing features [1].

Retroperitoneal cystic lymphangioma is a rare, benign mesodermal tumour arising from the retroperitoneal lymphatics. Lymphangiomas are histologically classified as capillary, cavernous and cystic with the retroperitoneal types being almost always cystic [2]. These cystic lesions may be unilocular or multilocular and contain serous or chylous fluid. The majority (more than 95\%) are situated in the head, neck, axilla and extremeties. Other sites including the abdomen are seen only in 5\% of cases [2]. The intraabdominal cystic lymphangiomas occur in less than 1 per 100,000 hospital admissions. Retroperitoneal lymphangiomas are rarer than abdominal lymphangiomas of mesenteric origin. The postulated mechanism for the formation of lymphangioma is the early developmental sequestration of lymphatic vessels that fail to establish connection with normal draining vessels at about 14 to 20 weeks of intrauterine life [2].

Due to its potential to grow, invade vital structures and develop life-threatening complications, complete laparoscopic excision should be considered as a therapeutic option to treat retroperitoneal cystic lymphangioma [3]. If surgical excision is used in treatment, it needs to be as complete as possible to reduce the risk of recurrence. Trindade et al excised retroperitoneal lymphangioma [3]. The last report of retroperitoneal lymphangioma was done by Ergul et al. [4] only 3 cases of lymphangioma located in the lesser sac have been reported in the literature. [5-7] Hiroaki Tsukada et al. reported a rare case of small bowel lymphangioma which was diagnosed with laparoscopy [8]. However, J.-Y. Choi et al. showed that a rare case of cavernous lymphangioma originating in the gallbladder. [9]

Outcomes following complete resection of retroperitoneal lymphangiomas are generally good. [3] Surgery is often required for symptom control or diagnosis [1]. Recurrence of symptoms with incomplete excision is possible. Dissemination in the retroperitoneum is very rare but potentially a fatal complication [1]. Hauser et al. suggested that isolation and ligation of the cystic lymphangioma's peduncle as well as ligation of lymph channels can prevent recurrences and chylascos. [10]

\section{Conclusions}

First of all, diagnostic tools in such cases are not able to support your differential diagnosis. In such circumstances surgeons and practitioners are recommended to consider the rare cases .Adult retroperitoneal lymphangioma is the rare case which can be diagnosed simply by laparoscopy.

\section{References}

[1] M. Cherk, M. Nikfarjam and C. Christophi, "Retroperitoneal Lymphangioma,” Asian Journal of Surgery, Vol. 29, 2006, pp. 51-54. doi:10.1016/S1015-9584(09)60297-9

[2] D. V. Rani, R. Srilakshmi, S. Malathi, V. Raghupathy and R. K. Bagdi, "Unusual Presentation of a Retroperitoneal Lymphangioma,” Indian Journal of Pediatrics, 2006: 73; 617-618. doi:10.1007/BF02759928 
[3] E. N. Trindade, M. R. Trindade, J. C. Boza, V. Von Diemen and R. B. Ilgenfritz, "Laparoscpic Excision of a Retroperitoneal Cystic Lymphangioma in an Elderly Patient," Minerva Chirurgica, 2007; Vol. 62, No. 2, pp. 145-147.

[4] E. Ergul, S. M. Sarikaya, B. Korukluoglu, A. E. Ucar and A. Kusdemir: "Retroperitoneal Cystic Lymphangioma," The Internet Journal of Surgery, 2008,15,1.

[5] G. Uggeri, M. Arcidiaco, U. Pandolfi, et al. "Cystic Lymphangioma of Lesser Epiploon (On a Case Brought to Our Attention)," Annali Italiani di Chirurgia, 1986, Vol. 56, pp. 159-170.

[6] Tezuka K, Ogawa Y, Satake K, et al. "Lymphangioma of Lesser Omentum Associated with Abdominal and Esophageal Carcinoma: Report of a Case,” Surgery Today, 2002; Vol. 32, pp. 362-366. doi:10.1007/s005950200053
[7] M. Ouaissi, O. Emungania, H. Sebbag, et al. "Cystic Lymphangioma of Lesser Omentum,” La Presse Médicale, 2003, Vol. 32, pp. 1165-1166.

[8] H. Tsukada, K. Takaori, S. Ishiguro, T. Tsuda, S. Ota and T. Giant "Cysti lymphangioma of the Small Bowel Mesentery: Report of a Case,” Surgery Today, 2002, Vol. 32, No. 8, pp. 734-737.

[9] J.-Y. Choi, M.-J. Kim, J.-J. Chung, S.-I. Park, J. T. Lee, H. S. Yoo, L. Kim and J.-S Choi, "Gallbladder Lymphangioma: MR Findings,” Abdominal Imaging, 2002, Vol. 27, No. 1.

[10] H. Hauser, H. J. Mischinger, A. Beham, A. Berger, H. Cerwenka, J. Razmara, H. Fruhwirth and G. Werkgartner "Cystic Retroperitoneal Lymphangiomas in Adults," European Journal Surgery Oncology, 1997, Vol. 23, pp. 322-326. doi:10.1016/S0748-7983(97)90777-0 